\title{
Scale-free properties of weighted networks with connectivity-driven topology
}

\author{
W. Jeżewski ${ }^{1}$ \\ Institute of Molecular Physics, Polish Academy of Sciences, Smoluchowskiego 17, \\ 60-179 Poznań, Poland
}

\begin{abstract}
The rate equations are used to study the scale-free behavior of the weight distribution in evolving networks whose topology is determined only by degrees of preexisting vertices. An analysis of these equations shows that the degree distribution and thereby the weight distribution remain unchanged when the probability rate of attaching new nodes is replaced with an unnormalized rate determined by the ratio of the degree of a randomly selected old node to the maximal node degree at the current stage of the network evolution. Such a modification of the attachment rule is argued to accelerate considerably numerical simulations of both unweighted and weighted networks belonging to the class of investigated evolving systems. It is also proved that the studied rate equations have a solution corresponding to the total weight (concentrated at individual vertices) distribution displaying the power-law behavior for asymptotically large weights.
\end{abstract}

PACS: $02.50 . \mathrm{Cw} ; 84.35 .+\mathrm{i} ; 87.23 . \mathrm{Ge} ; 89.75 . \mathrm{Hc} 4$

Key words: Weighted networks, Rate equations, Scaling

\section{Introduction}

Scale-free networks belong to an important group of complex evolving systems exhibiting a power-law of the degree (connectivity) distribution [1]. To explain the origin of the scaling form of the degree distribution, several scenarios of the growth and self-organization of networks have been proposed [2-11]. Probably the most natural mechanism responsible for scaling properties of time-evolving networks is the preferential attachment of a new vertex with the probability

1 e-mail: jezewski@ifmpan.poznan.pl 
being proportional to the degree of a randomly selected preexisting node to which a new one will be connected [2-4]. This simple self-organization mechanism allows one to recover the complex topological structure of scale-free networks $[1,3]$. However, in many real network systems, links perform some specific functions, and the underlying binary networks can only be considered as skeletons determining topological properties of these systems. Thus, in real networks, links play a role of arteries through which some processes of different intensities are executed, or which carry loads of different magnitudes [12-17]. Functional properties of networks can be characterized by assigning loads or weights to particular links [18]. Then, the network evolution is in general described by both preferential node attaching and assigning weights to links. Essentially, processes of the preferential node attachment and the link loading are not autonomous. The preferential mechanism of the vertex attachment can be determined only by degrees of existing vertices [18], but can also be affected by weights, or can even be controlled entirely by total weights concentrated at preexisting vertices [15-17]. On the other hand, weights ascribed to newly created links can depend on degrees of old nodes to which new nodes are connected [18], or, in cases when internal (existing) links are loaded, the weight assigned to a given internal link can be expressed by degrees of both vertices joined by this link [12]. Models in which the attachment rule is entirely governed by degrees of existing nodes appear to be appropriate for describing not only topological but also functional features of the WWW. In this network, the decision about linking a pair of nodes (webpages) depends on the popularity (measured by the node degree) of a given preexisting node to which an another node will be connected rather than on the weight concentrated at the existing node, i.e., the total size of documents sent from this node to others. Numerical simulations of networks with degree-driven topologies indicate that the total weight distribution can display in such systems a power-law form [18,19]. In turn, models whose topologies are entirely controlled by weights provide a representation of real systems in which a change of network traffic due to connecting a new node to an old node strongly disturbs functions performed by all links joining the old node with other nodes, or in which connections of new vertices influence functions of whole systems. Illustrative examples of such real networks are transportation systems [15-17]. It has been shown analytically that, in the case of linear dependence of the attachment probability on total weights concentrated at vertices, the distribution of total weights can also display a power-law form [15-17].

In this paper, undirected weighted networks with connectivity driven topologies are considered. By analyzing the rate equations for the degree and weight distributions in such networks, it is shown that these distributions remain unchanged as the preferential mechanism of node attaching is appropriately modified by using an unnormalized attachment rule. This change of the vertex attaching rule is shown to be very useful for numerical simulations of some unweighted (binary) networks as well as for simulations of the consid- 
ered weighted networks with degree-governed topological structures. The rate equations for the time evolution of the distribution of total weights concentrated at vertices of a given connectivity is proved to have a solution of a scale-free form.

\section{Evolution of the degree distribution in binary networks}

In this section, the preferential attachment mechanism that a new node is linked to a randomly chosen preexisting node with the probability depending

on the degree of the earlier node is revised. In general, this probability is assumed in the form $[4,20]$

$$
\Pi_{\gamma}(k)=A_{\gamma}(k) / M_{\gamma}(t)
$$

with the connection kernel

$$
A_{\gamma}(k)= \begin{cases}k^{\gamma} & , \gamma \neq 1 \\ a_{k} k & , \gamma=1\end{cases}
$$

and with the normalization factor $1 / M_{\gamma}(t)$ determined by

$$
M_{\gamma}(t)=\sum_{j=1}^{k_{\max }} A_{\gamma}(j) N_{j}(\gamma ; t)
$$

where $\gamma \geq 0$ is the nonlinearity exponent, $k$ denotes the vertex connectivity, $k_{\max }=k_{\max }(\gamma ; t)$ is the maximal node degree, the amplitudes $a_{k} \geq 0, k=$ $1,2, \ldots$, and $N_{k}(\gamma ; t)$ denotes the number of vertices of the degree $k$. Note that the power-law $N_{k} \sim k^{-\nu}$ emerges only if the kernel $A_{\gamma}(k)$ is linear or asymptotically linear, i.e., when $\gamma=1$ and the amplitudes $a_{k}$ are all equal, or are unequal, but such that $a_{k} \rightarrow a_{\infty}$ as $k \rightarrow \infty[20]$.

It is proved below that the degree distribution remains unchanged when the probability attachment rate $\Pi_{\gamma}(k)$ is replaced with the rate

$$
\tilde{\Pi}_{\gamma}(k)= \begin{cases}k^{\gamma} / k_{\max }^{\gamma}(\gamma ; t) & , \gamma \neq 1 \\ a_{k} k / a_{\infty} k_{\max }(1 ; t) & , \gamma=1\end{cases}
$$

For cases of $0 \leq \gamma \leq 1$, this modified attachment rate is unnormalized (thus, it does have the character of a probability rate), and is not proportional to 
the rate $\Pi_{\gamma}(k)$ as, in these cases, time dependences of $k_{\max }(\gamma ; t)$ and $M_{\gamma}(t)$ are different. If $a_{k}>a_{\infty}$ (for some $k<k_{\max }$ ), the rate $\tilde{\Pi}_{1}(k)$ takes even values greater than one. To show the equivalence of both preferential linking procedures, one can apply the rate equations describing, for the attachment rate (1), the time evolution of the degree distribution [20]

$$
\frac{d}{d t} N_{k}(\gamma ; t)=\frac{1}{M_{\gamma}(t)}\left[A_{\gamma}(k-1) N_{k-1}(\gamma ; t)-A_{\gamma}(k) N_{k}(\gamma ; t)\right]+\delta_{k, 1}
$$

Solutions of these equations imply for large $t$ that $[20,21]$

$$
M_{\gamma}(t)= \begin{cases}\mu t & , \quad 0 \leq \gamma \leq 1 \\ t^{\gamma}, & \gamma>1\end{cases}
$$

where $\mu$ is a function of $\gamma$, such that, in cases of homogeneous kernels with uniform, unit amplitudes, $\mu \in[1,2]$ for all $\gamma \geq 0(\mu=1$ for $\gamma>1)$, and

$$
k_{\max }(\gamma ; t)= \begin{cases}\left(\frac{1-\gamma}{\mu}\right)^{\frac{1}{1-\gamma}}(\ln t)^{\frac{1}{1-\gamma}} & , 0 \leq \gamma<1 \\ s(\nu) t^{\frac{1}{\nu-1}} & , \gamma=1 \\ t & , \gamma>1\end{cases}
$$

with $\nu \geq 2, s$ being dependent on $\nu$ as well as on the manner of the convergence of $a_{k}$ to $a_{\infty}$ as $k \rightarrow \infty$ (in the case of $\gamma=1$ ). Since, $k_{\max }(\gamma ; t)$ is a monotonic function of time for each $\gamma$, Eq. (5) can be transformed from $t$ to $k_{\max }$ using the inverse function $t=g_{\gamma}\left(k_{\max }\right)$. Thus, the use of Eqs. (6) and (7) gives

$$
M_{\gamma}(t)=k_{\max }^{\gamma} g_{\gamma}^{\prime}\left(k_{\max }\right)
$$

where, $g_{\gamma}^{\prime}$ denotes the derivative of $g_{\gamma}$ with respect to $k_{\max }$. Next, inserting (8) into Eq. (5), replacing $t$ by $k_{\max }$, and multiplying these equations by $g_{\gamma}^{\prime}\left(k_{\max }\right)$, one obtains

$$
\begin{aligned}
& \frac{d}{d k_{\max }} \tilde{N}_{k}\left(\gamma ; k_{\max }\right)= \\
& \frac{1}{k_{\max }^{\gamma}}\left[\tilde{A}_{\gamma}(k-1) \tilde{N}_{k-1}\left(\gamma ; k_{\max }\right)-\tilde{A}_{\gamma}(k) \tilde{N}_{k}\left(\gamma ; k_{\max }\right)\right]+\delta_{k, 1} g_{\gamma}^{\prime}\left(k_{\max }\right)
\end{aligned}
$$

with $\tilde{N}_{k}\left(\gamma ; k_{\max }\right) \equiv N_{k}\left(\gamma ; g_{\gamma}\left(k_{\max }\right)\right), \tilde{A}_{\gamma}(k) \equiv A_{\gamma}(k)$ for $\gamma \neq 1$ and $\tilde{A}_{1}(k)=$ $\left(a_{k} / a_{\infty}\right) A_{1}(k)$. In Eq. (9), the role of time is played by the variable $k_{\max }$. The factor $g_{\gamma}^{\prime}$ in the last term on the right hand side of (9) determines for 
$0 \leq \gamma \leq 1$ the acceleration of introducing new nodes (of degree one), as $k_{\text {max }}$ increases (the number of new nodes introduced in successive equal intervals of $k_{\max }$ is for $0 \leq \gamma \leq 1$ a growing function of $\left.k_{\max }\right)$. Note that $g_{\gamma}^{\prime}\left(k_{\max }\right)=1$ for $\gamma>1$. The rate equation (9) can be solved using the substitutions (cf. Ref. 20) $\tilde{N}_{k}\left(\gamma ; k_{\max }\right)=n_{k} g_{\gamma}\left(k_{\max }\right)$ for $0 \leq \gamma \leq 1$ and $\tilde{N}_{k}\left(\gamma ; k_{\max }\right)=J_{k} g_{\gamma}^{k-(k-1) \gamma}\left(k_{\max }\right)$ for $\gamma>1$. Thus, according to (7), the systems of equations (5) and (9) are equivalent and, consequently, solutions to them yield the same degree distribution. However, instead of the original attachment rate, determined by Eqs. (1)-(3), the rate occurring in Eq. (9) is of the form (4). These rates satisfy for $\gamma \leq 1$ the inequality $\tilde{\Pi}_{\gamma}(k)<\Pi_{\gamma}(k), k=1,2, \ldots$, except for cases of some $a_{k}<a_{\infty}$ (when $\gamma=1$ ), for which the inequality holds only for sufficiently large $k$. Therefore, using the preferential rate (4) in numerical simulations of evolving binary networks enables one to speed up computations, with regard to analogous computations based on employing the rate (1), for the same exponent $\gamma \leq 1$ and for the same set of amplitudes $\left\{a_{k}\right\}$ (when $\gamma=1$ ). The speed up ratio of computations at a given stage of the network generation (i.e. at a given moment $t$ ) is determined by the quotient of the attachment probabilities (4) and (1), or by the quotient of $M_{\gamma}(t)$ and $k_{\max }^{\gamma}(\gamma ; t)$. By means of $(8)$, this momentary ratio is given by $\tilde{\tau}_{\gamma}(t)=g_{\gamma}^{\prime}\left(k_{\max }(\gamma ; t)\right)$. Clearly, $\tilde{\tau}_{\gamma}(t)$ is equal to the evolution acceleration factor occurring in the last term on the right hand side of Eq. (9). The total speeding up ratio $\tau_{\gamma}(N)$, determining the reduction of the entire period of time during which a network is numerically generated, beginning with one node at the initial time $t_{i}=1$ and ending with $N$ nodes at the final time $t_{f}=N$, can be estimated using the relation

$$
\tau_{\gamma}(N)=(N-1)^{-1} \int_{1}^{N} g_{\gamma}^{\prime}\left(k_{\max }(\gamma ; t)\right) d t
$$

By applying (7), one finds for $0 \leq \gamma \leq 1$ and for large $N$ that

$$
\tau_{\gamma}(N) \sim g_{\gamma}^{\prime}\left(k_{\max }(\gamma ; N)\right)
$$

and hence

$$
\tau_{\gamma}(N) \sim \begin{cases}(\ln N)^{-\frac{\gamma}{1-\gamma}} N & , 0 \leq \gamma<1 \\ N^{\frac{\nu-2}{\nu-1}} & , \gamma=1\end{cases}
$$

Notice that, in the case of $\gamma>1$, Eqs. (10) and (7) imply that $\tau_{\gamma}(N)=$ 1. Because $\tau_{\gamma}$ is an increasing function of $N$ for each $\gamma$ from the interval $[0,1]$, the application of the rate (4) in numerical calculations in cases of $0 \leq \gamma \leq 1$ becomes more and more effective as $N$ grows (compared with analogous calculations with the use of the rate (1)). Clearly, this is of rather 
little importance, as Eq. (5) are exactly solvable for all $k$ when $\gamma=1$ and for asymptotically large $k$ when $0 \leq \gamma \leq 1$. It turns out, however, that, in simulations of some networks with more complex normalized linking rules than given by Eq. (1), the replacement of normalized attaching rate with an unnormalized rate of the type (4) is also possible [22]. Furthermore, the application of the linking rate (4) can be very useful for numerical simulations of weighted networks with connectivity-driven topologies, especially in cases when the weight distribution cannot be found exactly.

Time needed to simulate networks evolved by node attaching with the probability rate with the strictly linear kernel can also be reduced by using, instead of the attachment rate (1), a uniform initial attachment rate (corresponding to $\gamma=0$ ), combined with the redirection, with probability $r=0.5$, of newly created links to ancestors of selected preexisting nodes [21]. Another way to enhance the efficiency of simulations of growing networks is the application of parallel algorithms [23]. Although by using different evolution rules one can generate in the large $N$ limit networks revealing the same (at least for large connectivities) degree distribution, these networks can exhibit different histories of growth. However, the statistics of weights introduced in such connectivity-driven networks cannot necessarily be independent of histories of the network growth. This can happen when loads are ascribed to links as soon as the links are created, and when loads magnitudes depend strongly on details of network histories. Consequently, weighted networks can display different weight distributions, even if they undergo the same rule of link loading and even if they exhibit the same degree distribution. Therefore, the rate equation approach to study the weight distribution in networks with connectivity-governed topologies should, in general, incorporate rate equations for the evolution of the degree distribution.

\section{Evolution of weighted networks}

The process of link loading can be carried out in many different ways, according to various functions performed by real networks [15-19]. For example, to mimic functional properties of transportation or communication networks, an evolving model has been elaborated by assuming that the topology of the underlying skeleton network is entirely determined by total weights concentrated at vertices to which new vertices are connected, and by assuming that the procedure of ascribing a weight to a newly created link involves a redistribution (perturbation) of weights, assigned to all links concentrated at the existing vertex to which new vertex has been connected [15-17]. However, there exist also real networks whose topologies are governed by vertex degrees only. Perhaps the most representative example of such networks is the WWW. The appearance of a new link in this network depends on the popularity of a given 
node (webpage) that connects a new node rather than depends on the node weight (the total size of documents taken from the preexisting webpage). Naturally, the popularity of a given webpage can be measured by its connectivity (the number of directed hyperlinks joining this webpage with other webpages). Although traffic jams in the WWW reduce the speed of sending documents from one webpage to others, the total size (usually unknown) of documents sent from a given webpage has no effect on the mere act of creating a hyperlink between this webpage and another one. Thus, the topology of the WWW can be considered as independent of weights assigned to links.

Here, weighted networks in which node attaching is determined by the probability rate (1) (or by the rate (4)) are studied. For simplicity, only the linear case $\gamma=1, a_{k}=1, k=1,2, \ldots$, of the preference rate is discussed. Each of the links $i \leftrightarrow j$ of a given network is taken to be loaded by a weight $w_{i, j}>0$, and, consequently, each vertex is assumed to concentrate a total weight $w_{i}=\sum_{j} w_{i, j}$. The number of nodes with $k$ links and the total weight $w$ is given by

$$
N_{k}(w, t)=\sum_{i=1}^{N_{k}(t)} \delta\left(w-w_{i}\right)
$$

where the sum runs over all $N_{k}(t)$ nodes with $k$ links and $\delta$ denotes the Dirac delta function. The rate equations for the evolution of the number $N_{k}(w, t)$ of nodes with $k$ links and with the total weight $w$ can be expressed in the form

$$
\begin{aligned}
& \frac{\partial}{\partial t} N_{k}(w, t)= \\
& \quad \frac{k-1}{M(t)} \int_{\varepsilon_{k-1}}^{w-E_{k-1}} \rho_{k-1}(x) N_{k-1}(w-x, t) d x-\frac{k}{M(t)} N_{k}(w, t)+ \\
& \quad \delta_{k, 1} \rho_{k}\left(w-E_{k}+\varepsilon_{k}\right),
\end{aligned}
$$

where $M(t) \equiv M_{1}(t)=\sum_{j} j N_{j}(t), \varepsilon_{k-1}$ is the minimal weight that can be introduced to any vertex with $k-1$ links in consequence of connecting a new link, $E_{k-1}$ denotes a minimal of total weights concentrated at vertices of the degree $k-1$, and $\rho_{k}(x)$ is the probability distribution of weights that can be assigned to a new link connected to any of vertices of the degree $k$. Similarly to the limit weights $\varepsilon_{k}$ and $E_{k}$, the distribution $\rho_{k}(x)$ is determined by rules which govern the weight loading [19]. The first term on the right hand side of Eq. (14) is responsible for different processes of the increase of total node weights from the value $w-x$ to $w$, as a result of connecting new links with weights $x \in\left[\varepsilon_{k-1}, w-E_{k-1}\right]$ (note that $w \geq E_{k-1}+\varepsilon_{k-1}=E_{k}$ ). The second and third terms in (14) are the loss and the new site terms, respectively [20]. Obviously, integrating Eq. (14) over all $w$ one recovers the rate equation (5) 
for the connectivity distribution, in the case of the linear connection kernel. In accordance with the argumentation presented in the previous section, the evolution of $N_{k}(w, t)$ can alternatively be investigated with respect to $k_{\max }$.

Since $M(t)=2 t[20]$, one can substitute $N_{k}(w, t)=t n_{k}(w)$. Hence, using (14), one obtains

$$
\begin{aligned}
n_{k}(w)= & \frac{k-1}{k+2} \int_{\varepsilon_{k-1}}^{w-E_{k-1}} \rho_{k-1}(x) n_{k-1}(w-x) d x+ \\
& \frac{2}{k+2} \delta_{k, 1} \rho_{k}\left(w-E_{k}+\varepsilon_{k}\right) .
\end{aligned}
$$

To show that there exists for large $w \gg \varepsilon_{k}, E_{k}$ a solution to Eq. (15) of the scaled form $n_{k}(w) \sim w^{-\alpha}, \alpha>1$, assume that

$$
n_{k}(w) \simeq n_{k} c_{k}(\alpha) w^{-\alpha}
$$

where

$$
n_{k}=\frac{4}{k(k+1)(k+2)}
$$

is the exact solution of the rate equations for the connectivity distribution in the case of the linear connection kernel [20], and $c_{k}(\alpha)$ is the normalization constant, determined by the condition

$$
c_{k}(\alpha) \int_{E_{k}}^{\infty} x^{-\alpha} d x=1 .
$$

The scaling expression (16) refers to cases when a weight $w$ is assigned to each link with probability determined for all $k$ by the same tail contribution $\rho(w)$ to the distribution $\rho_{k}(w)$, i.e. $\rho_{k}(w) \simeq \rho(w)$ for $w \gg 1$, where

$$
\rho(w)=\lambda w^{-\alpha}
$$

with $\lambda>0$ being independent of $w$ and equal for all $k$. Consequently, substituting (16) into (15), using Eq. (17) and employing the condition (18), one finds for asymptotically large $w$ that

$$
\left(1+\frac{\varepsilon_{k-1}}{E_{k-1}}\right)^{\alpha-1}=1+\left(\frac{\varepsilon_{k-1}}{E_{k-1}}\right)^{\alpha-1}+O\left(\frac{1}{w}\right)
$$


where the relation $E_{k}=E_{k-1}+\varepsilon_{k-1}$ has been applied. Eq. (20) holds if $\alpha=2$ (for $w \rightarrow \infty$ ). Thus, in the case of $\alpha=2$, the scaling function (16) indeed satisfies Eq. (15) when $w$ tends to infinity. Accordingly, the total weight distribution $P(w)=\sum_{k} n_{k}(w)$ undergoes for asymptotically large $w$ the power-law

$$
P(w) \simeq b w^{-2}
$$

where the constant $b=\sum_{k} n_{k} c_{k}(\alpha)$. By means of (17) and (18), this constant is finite when $k^{-2} E_{k} \rightarrow 0$ as $k \rightarrow \infty$. The weight distribution has also been shown to display a power-law behavior in cases of networks in which the growth is governed by homogeneous and inhomogeneous attachment rules depending linearly on the degree of the existing node that connects new node, and in which links receive weights being powers of products of degrees of joined nodes $[24]$.

The above analysis shows that, in networks with connectivity-driven topology and with a special distribution $\rho_{k}(x)$ of weights assigned to links, the rate equations for the time evolution of the distribution of weights concentrated at vertices with a given connectivity have, for asymptotically large weights, a solution of the scale-free form. This solution refers to a simple case when the numbers of vertices of different degrees are all subjected to the power-law for the weight with the same scaling exponent $\alpha$. Thus, the solution to the considered rate equations possesses the power-law form with the same scaling index also in cases when the preference rate of attaching vertices is not linear [20], provided that the distribution of weights assigned to links is of the form (19). The question of whether the studied rate equations for the total weight distribution have also other solutions (in cases of other distributions $\rho_{k}(x)$ than the one considered above), for which the total weight distribution $P(w)$ would reveal a scaling form at large $w$, is open. Clearly, what values could take the scaling index describing the power-law decay of the total weight distribution, or whether this distribution could undergo the power-law at all, it would depend on specific rules of link loading in an individual network. Note that the rate equation approach has been applied to investigate weight and degree distributions in networks with weight-driven topologies [25].

\section{References}

[1] R. Albert, A.-L. Barabási, Rev. Mod. Phys. 74 (2002) 47.

[2] A.-L. Barabási, R. Albert, Science 286 (1999) 509.

[3] A.-L. Barabási, R. Albert, H. Jeong, Physica A 272 (1999) 173.

[4] A.-L. Barabási, R. Albert, H. Jeong, Physica A 281 (2000) 69. 
[5] B.A. Huberman, L.A. Adamic, Nature 401 (1999) 131.

[6] K.-I. Goh, B. Khang, D. Kim, Phys. Rev. Lett. 88 (2002) 108701.

[7] S.N. Dorogovtsev, J.F.F. Mendes, Phys. Rev. E 62 (2000) 1842.

[8] S.N. Dorogovtsev, J.F.F. Mendes, A.N. Samukhin, Phys. Rev. E 63 (2001) 062101.

[9] S.N. Dorogovtsev, A.V. Goltsev, J.F.F. Mendes, Phys. Rev. E 65 (2002) 066122.

[10] A. Vázquez, cond-mat/0006132.

[11] K. Klemm, V.M. Eguíluz, Phys. Rev. E 65 (2002) 036123.

[12] M.E.J. Newman, Phys. Rev. E 64 (2001) 016131; 016132.

[13] M.E.J. Newman, cond-mat/0407503.

[14] S. Maslov, V.-C. Zhang, Phys. Rev. Lett. 87 (2001) 248701.

[15] A. Barrat, M. Barthélemy, A. Vespignani, Phys. Rev. Lett. 92 (2004) 228701.

[16] A. Barrat, M. Barthélemy, R. Pastor-Satoras, A. Vespignani, Proc. Natl. Sci. USA 101 (2004) 3747.

[17] A. Barrat, M. Barthélemy, A. Vespignani, cond-mat/0406238.

[18] S..H. Yook, H. Jeong, A.-L. Barabási, Phys. Rev. Lett. 86 (2001) 5835.

[19] W. Jeżewski, Physica A 337 (2004) 336.

[20] P.L. Krapivsky, S. Redner, F. Leyvraz, Phys. Rev. Lett. 85 (2000) 4629.

[21] P.L. Krapivsky, S. Redner, Phys. Rev. E 63 (2001) 066123.

[22] W. Jeżewski, Phys. Rev. E 66 (2002) 067102.

[23] B. Machta, J. Machta, cond-mat/0408372.

[24] E. Almaas, P.L. Krapivsky, S. Redner, cond-mat/0408295.

[25] T. Antal, P.L. Krapivsky, cond-mat/0408285. 